\title{
¿El trabajo es salud?
}

Is Work Healthy?

\section{María Luz Vega Ruíz}

1Departamento de Investigación de la Organización Internacional del Trabajo, Ginebra, Suiza.

Fechas · Dates

Recibido: 2020.02 .26

Aceptado: 2020.03.11

Publicado: 2020.11.04
Correspondencia $\cdot$ Corresponding Author

María Luz Vega Ruíz

Departamento de Investigación de la Organización Internacional del Trabajo vega@ilo.org 
Trabajo y salud aparecen prima facie como conceptos diferentes, aunque se trata de "hechos" intrínsecamente interrelacionados, por cuanto hoy es impensable que el trabajo pueda hacerse en condiciones adecuadas en un estado de ausencia de salud. De hecho, para la OMS la salud "es un estado de completo bienestar físico, mental y social, y no solamente la ausencia de afecciones o enfermedades".(1) Esta definición confirma, la clara interrelación entre dos hechos en principio diferentes, pero en la práctica interdependientes: la salud es imprescindible para trabajar y las condiciones en las que se realiza el trabajo pueden perjudicar la salud.

Nadie duda hoy que la Revolución Industrial y como consecuencia una nueva forma de trabajar, ocasionó cambios sustantivos en las condiciones de vida y bienestar de los trabajadores, razón por la cual diversos investigadores de perfil social o económico, comenzaron a estudiar el impacto de los cambios industriales sobre la salud de la población trabajadora. Marx y Engels, Villerme, Guérin, Penot y Benoiston de Chateauneuf(2), son algunos de los que consideraban el deterioro de la salud como uno de los efectos del capitalismo en los nuevos métodos de trabajo,

En la actualidad nadie duda que las consecuencias sociales del modelo económico de desarrollo existente, además de, producir pobreza, desempleo, y merma en la calidad del empleo, han afectado de forma negativa a la salud de los trabajadores, incrementando el número de accidentados y enfermos ocupacionales. La OIT calcula 7.500 muertos por día como consecuencia de ambientes de trabajo inseguros e insalubres, de los cuales 6.500 fallecen por causa de enfermedades contraídas en el trabajo. ${ }^{(3)}$

Cada día mueren personas a causa de accidentes laborales o enfermedades relacionadas con el trabajo - más de 2,78 millones de muertes por año. Además, anualmente ocurren unos 374 millones de lesiones relacionadas con el trabajo no mortales, que resultan en más de 4 días de absentismo laboral ${ }^{(3)}$. El coste de esta adversidad diaria es enorme y la carga económica de las malas prácticas de seguridad y salud se estima en un 3,94 por ciento del Producto Interior Bruto global de cada año. ${ }^{(3)}$ Es evidente considerar que la salud es parte esencial del estudio del trabajo y está en el origen del desarrollo social, y que es necesario contar con un trabajo decente y un medio ambiente saludable

Paralelamente, y como ya se esbozó, en el mundo del avance de la ciencia médica y del uso del algoritmo en el tratamiento personalizado del enfermo, el trabajo aparece hoy más que nunca en el origen de nuevos riesgos, enfermedades e incluso como elemento de distorsión de la salud pública. ¿Cómo es posible este cambio?

La protección de la salud, que fue parte de las primeras leyes sociales, es hoy causa de preocupación en un mundo donde la actividad profesional se ve afectada por elementos externos de riesgo, como la polución medioambiental y la invasión tecnológica, e internamente por los cambios en los sistemas de producción, por la necesidad de nuevas competencias laborales y, en fin, por una gestión diferente de los llamados recursos humanos.

Como resultado, según el estudio de The Work Force View ${ }^{(4)}$, en 2018, una de cada cinco personas en Europa (18\%) afirmaba sufrir de estrés todos los días y un $30 \%$ 
de ellas se sentían tan estresadas que se planteaban cambiar de trabajo. No obstante, el $79 \%$ de los entrevistados se consideran optimistas, lo que genera aún más contradicciones al intentar interpretar los resultados. ¿Un futuro de optimistas estresados? Esta es sin duda una conclusión simplista.

Trabajar sigue siendo hoy una necesidad y una forma de realización humana, aunque por sus condiciones actuales sea también una causa importante de estrés y de enfermedades, que amplían su espectro más allá de los riesgos profesionales conocidos. El reciente reconocimiento del burn out por la OMS como enfermedad, y la inclusión de los trastornos mentales y del comportamiento en la lista de enfermedades profesionales de la OIT(5), constatan el hecho de que la salud del trabajador enfrenta en el siglo XXI retos invisibles que van más allá de lo conocido, de lo predecible y de lo que con los medios actuales es posible prevenir.

Es innegable que el mundo de hoy ofrece formas de trabajo muy distintas en contenido y condiciones de las existentes después de la primera revolución industrial. Hoy se plantean dicotomías tales como la autonomía del trabajador versus la inestabilidad, la necesidad de capacidades y formación especiales versus la tendencia a un menor esfuerzo físico, la disminución de la penosidad y el impulso de la creatividad versus el trabajo mecánico y repetitivo, y la deslocalización del centro de trabajo por una mayor independencia y flexibilidad versus la sociedad 24 horas/ 7 días.

En este contexto, la necesidad de preservar la salud mental y el equilibrio personal adquiere preminencia sobre la defensa de la salud física de forma exclusiva, lo que ilustra la permeabilidad mayor de lo laboral a todas las esferas sociales y humanas. Es decir, el trabajo se expande por todos los ámbitos humanos, convirtiendo la salud en un bien de obligada protección. Se siguen necesitando hoy trabajadores "sanos", pero se concibe el bienestar social como una aspiración universal. No cabe trabajar sin tener salud.

La salud laboral aparece hoy estrechamente ligada a la salud pública, ya que, por ejemplo, normas medioambientales en materia de contaminación del aire, tienen un impacto directo en las normas de seguridad y producción en la empresa, y que cualquier forma de violencia social afecta el mundo del trabajo.

La salud laboral no quedará circunscrita a la limitación de la penosidad o la peligrosidad de la tarea, que se verá aliviada por el uso de la máquina, sino por el nivel de concentración, estrés, sofisticación y adaptación al cambio que pueden llevar consigo las nuevas formas de trabajo, así como los efectos de un cambio climático que puede trasformar radicalmente el trabajo.

Nuevas formas de trabajo a distancia, provocan nuevos conflictos y situaciones de stress. De hecho, el $41 \%$ de los trabajadores con trabajo móvil basado en las TIC registran altos niveles de estrés, frente al $25 \%$ de los trabajadores que desempeñan sus tareas en las instalaciones del empleador ${ }^{(5)}$. Esto reviste especial importancia cuando los trabajadores tienen que trabajar desde casa más horas de las que les corresponden. El teletrabajo y el trabajo móvil basado en las TIC 
también se asocian a los trastornos del sueño que, a su vez, se relacionan con los niveles de estrés

La utilización de nuevos entes "automáticos" genera diferentes riesgos físicos y psíquicos, e incluso una nueva categoría de riesgos morales, al enfrentarse a problemas éticos. Las llamadas "enfermedades del progreso" son una nueva perspectiva que parece re-enfocar la prevención, basculando de lo físico a lo mental, y eso implica nuevas reglas y límites. Prueba de ello son las regulaciones sobre violencia y acoso en el trabajo, que han dado lugar incluso a la adopción de un nuevo Convenio internacional en el trabajo en la última Conferencia de la OIT ${ }^{(6)}$.

Desde la perspectiva actual, la salud adquiere un papel central en una sociedad donde la ecuación espacio/tiempo se diluye en el ejercicio de la actividad profesional. La necesidad de garantizar equilibrio entre la vida personal y profesional va más allá de avalar las condiciones de trabajo establecidas, ya que debe reflejar los ritmos biológicos de descanso necesario, lo que resulta hoy más complicado con el uso de nuevas tecnológicas y de "maquinas inteligentes" que permiten acceder a la vida personal y profesional simultáneamente (teléfonos móviles, tabletas, etc.). Más allá de la tecnología, el ser humano enfrenta un mundo "sucio", contaminado, donde respirar o beber agua limpia resulta difícil, a veces imposible.

Obviando versiones catastrofistas sobre el futuro, el trabajo debe seguir siendo fuente de salud, ya que es una aspiración humana. La idea de un nuevo (o revitalizado) contrato social aflora en las discusiones de las organizaciones nacionales e internacionales y no puede ser ajena. los temas relativos a la salud, por cuanto hablamos del eje de la vida. La salud es, por tanto, un derecho básico en todos sus ámbitos y es por ello en interés de la OIT en considerarlo como derecho fundamental en el trabajo.

Hoy, como siempre, el hombre y la mujer buscan paz y bienestar y un modus vivendi que satisfaga su esencia. Un mundo limpio y una vida saludable. Sólo el ser humano puede decidir sobre su futuro y, utilizando los medios a su alcance, seguir manteniendo las prioridades que orientaron la paz y el progreso en los últimos cien años. Por ello, aunque quizás con otro sentido, debamos seguir aseverando que el trabajo es salud y que no cabe hablar del futuro del trabajo sin hablar de la salud del futuro.

\section{Referencias}

1. Organización Mundial de la Salud. Preámbulo. Constitución de la Organización Mundial de la Salud. 1946 [cited 2020 Feb 23]. Disponible en: https://www.who.int/ governance/eb/constitution/es/

2. Yanes L. El trabajo como determinante de la salud. Salud de los Trabajadores. 2003;11(1):21-42.

3. Organización Internacional del Trabajo. Seguridad y salud en el centro del futuro del trabajo. Aprovechar 100 años de experiencia. Ginebra: OIT; 2019 [cited 2020 
Feb 23]. Disponible en: https://www.ilo.org/wcmsp5/groups/public/--ed_protect/--protrav/---safework/documents/publication/wcms_687617.pdf

4. ADP. The Workforce View in Europe 2018. 2018 [cited 2020 Feb 23]. Disponible en: https://www.en.adp.ch/assets/vfs/Domain-3/Workforce-View-2018/CH/ADPWorkforce-View-2018-SW-UK.pdf

5. Organización Internacional del Trabajo. Recomendación sobre la lista de enfermedades profesionales y el registro y notificación de accidentes del trabajo y enfermedades profesionales, 2002 (num 194). Ginebra: OIT; 2002 [cited 2020 Feb 23]. Disponible en: https://www.ilo.org/dyn/normlex/es/f?p=NORMLEXPUB:12100:0::NO:12100:P12100_INSTRUMENT_ID:312532:NO

6. Organización Internacional del Trabajo. Convenio sobre la violencia y el acoso, 2019 (núm. 190) [Internet]. Ginebra: OIT; 2019 [cited 2020 Feb 23]. Disponible en: https://www.ilo.org/dyn/normlex/es/f?p=1000:12100\%:NO:12100:P12100_INSTRUMENT_ID:3999810 